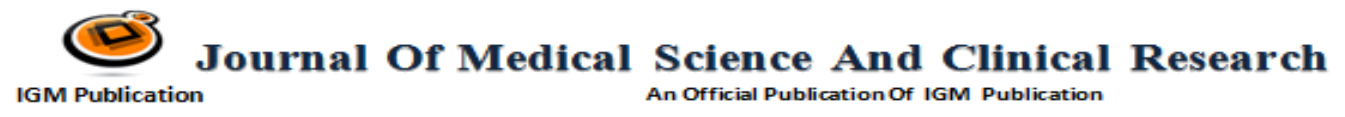

\title{
Hyperreflexic Guillain - Barre Syndrome: A Rare Case Report and a Brief Review of Literature
}

\author{
Authors \\ Dr Vajed Mogal ${ }^{1}$, Dr Sandeep Sanap ${ }^{2}$, Dr Pratik Patil ${ }^{3}$, Dr Preetam Ahire ${ }^{4}$ \\ ${ }^{1}$ Associate Consultant-Intensivist, MD (General Medicine) at Wockhardt Hospital, Nashik Maharashtra, \\ India - drvajedmogal@gmail.com 09822961158 \\ ${ }^{2}$ Senior Resident, MD (Medicine), M.G.M. Medical College, Aurangabad, Maharashtra \\ ${ }^{3,4}$ Junior Resident, Department of Medicine, MGM Medical College, Aurangabad, Maharashtra
}

\begin{abstract}
Guillain-Barre syndrome $(G B S)$ is a common cause of acute peripheral neuropathy and is characterized by hyporeflexia or areflexia. Hyperreflexia has been rarely reported with acute motor axonal neuropathy. A 65 year-old-female, known case of hypertension with type 2 diabetes mellitus, presented with asymmetrical weakness of upper and lower limbs and slurring of speech. Weakness progressed in the hospital with involvement of multiple cranial nerves, preserved deep tendon jerks with extensor plantar, and normal abdominal reflexes. She was treated with IV immunoglobulin and IV methylprednisolone. She was able to walk with support with normal voice at the time of discharge. This case reports highlights hyperreflexic guillain-barre syndrome as a rare presentation.
\end{abstract}

Key Words: Guillain-Barre syndrome(GBS), Quadriparesis, Hyperreflexia, Acute motor axonal neuropathy (AMAN)

\section{INTRODUCTION}

Guillain-Barre syndrome (GBS) is a neurological emergency that warrants prompt diagnosis and treatment. Guillain- Barré syndrome (GBS) is an acute autoimmune polyradiculoneuropathy, presenting as areflexic, flaccid paralysis with variable sensory disturbances, and elevated cerebrospinal fluid (CSF) protein without pleocytosis $^{1}$. The 2 features essential for a diagnosis of GBS are progressive motor weakness and areflexia. There are 2 distinctive pathologic subtypes of GBS: demyelinating and axonal. Recently, there have been several descriptions of reflex preservation and hyperreflexia in axonal GBS in Chinese, Japanese, and European populations $^{2}$. Although this variant is not common in the Indian subcontinent, a few cases have been reported $^{3,4}$. A high index of suspicion is needed to diagnose this rare presentation of GBS. We report a case of GBS with hyperreflexia which is a rare presentation.

\section{CASE REPORT}

A 65 year- old-female, known case of hypertension with type 2 diabetes mellitus on regular treatment, presented with pain and 
weakness in both upper and lower limbs more on the right side and of the distal muscles with inability to stand associated with slurring of speech since 3 days. She had history of fever with abdominal pain and diarrhoea lasting for 2 days, which improved with symptomatic treatment. Over the next 4 days, she had progressive weakness of limbs. At admission she had asymmetrical weakness of both upper and lower limbs and in the hospital her weakness progressed with involvement of shoulder muscles and cranial nerve involvement (bilateral weakness of facial muscles) with slurred speech. There was no other antecedent illness or recent vaccination. On examination, she was conscious, follows simple verbal commands with pulse rate of $82 / \mathrm{min}$, blood pressure of $140 / 80 \mathrm{~mm}$ of $\mathrm{Hg}$, respiratory rate of $18 /$ min and oxygen saturation was $98 \&$ on room air. On neurological examination there was bilateral 7 nerve, 10,11 , and 12 cranial nerve deficits, generalized hypotonia, and preserved deep tendon jerks with extensor plantar and normal abdominal reflex. There was no sensory deficit or autonomic dysfunction. Othr systemic examination were unremarkable.

Laboratory evaluation revealed normal total and differential leukocyte counts, erythrocyte sedimentation rate $(9 \mathrm{~mm} / \mathrm{h})$, and serum electrolytes ( $\mathrm{Na}, \mathrm{Ca}, \mathrm{K}, \mathrm{Mg}$, phosphate). Kidney $\&$ liver function tests were normal. Vasculitis work- up was negative. Thyroid function tests were normal. Serology for leptospirosis, HIV, Hepatitis $\mathrm{B}$ and $\mathrm{C}$ were negative. CSF examination showed 2 cells (100\% lymphocytes). CSF sugar was $74 \mathrm{mg} / \mathrm{dL}$ (plasma glucose 121 $\mathrm{mg} / \mathrm{dL}$ ) and protein was $68 \mathrm{mg} / \mathrm{dL}$. Nerve conduction study was suggestive of motor axonal polyneuropathy.

She was treated with intravenous immunoglobulin (IVIG) $(400 \mathrm{mg} / \mathrm{kg} /$ day) for 5 days and in view of progression of disease even after 2 days of IVIG therapy she was also treated with IV methylprednisolone. She recovered slowly with improvement of muscle power and was able to walk with support. At discharge 2 weeks later, she had a significant improvement and upper limb power was normal and lower limb power was $4 / 5$ proximally and 3/5 distally. She was able to walk with support and was under regular follow up. This case report highlights a rare presentation of GBS with hyperreflexia.

\section{DISCUSSION}

Guillain-Barré Syndrome (GBS) is an acute demyelinating disorder of the peripheral nervous system that results from an aberrant immune response directed at peripheral nerves. The term Guillain-Barré syndrome (GBS) includes a set of clinical syndromes with a common pathophysiological basis; an acute inflammatory polyneuropathy with a possible autoimmune etiolog 5 . Average annual incidence is 1.7 per $100,000^{6}$. A typical GBS patient presents with rapidly ascending symmetrical weakness, which may progress to respiratory Failure in $30 \%$ of patients ${ }^{7}$.

GBS is a group of syndromes with several distinctive subtypes classified on a pathologic basis into demyelinating and axonal forms. Axonal GBS has been classified further into 2 groups: AMAN and acute motor and sensory axonal neuropathy ${ }^{3}$. Although hyporeflexia or areflexia is the hallmark of GBS, normal reflexes or hyperreflexia is not a finding inconsistent with the diagnosis of GBS. The variants most commonly reported to be associated with retained or brisk reflexes are AMAN, acute motor conduction block neuropathy, and acute facial diplegia with brisk reflexes ${ }^{8,9}$. The incidence of hyperreflexia in AMAN is reported to be between $33 \%$ and $48 \%^{1,2}$.

Hyperreflexia is seen in GBS associated with antecedent C. jejuni infection; most of them have history of abdominal pain and diarrhea. These cases are usually mild and significant bulbar or respiratory involvement is uncommon. CSF analysis shows albuminocytologic dissociation in most cases. Almost all of them have IgG antiGM1 ganglioside antibodies although anti-C.Jejuni antibodies are frequently negative ${ }^{1}$. Antibody 
testing is not freely available in developing countries, such as India, which makes the diagnosis more difficult.

Normal reflexes or hyperreflexia throughout the course of GBS is unusual. Deep tendon reflexes may be preserved throughout the disease course in patients with acute motor axonal neuropathy (AMAN) and have been considered as indicators of rapid clinical recovery. Although hyperreflexia is a controversial symptom in patients with GBS, these findings indicate that there is functional corticospinal tract involvement in patients with a GBS variant. Moreover, $48 \%$ of Chinese and 33\% of Japanese patients with AMAN showed hyperreflexia in the recovery phase ${ }^{1,2}$. In another study, patients with pure motor GBS had preserved tendon reflexes up to MRC grade 3 paresis $^{10}$. Acute facial diplegia with hyperreflexia has been described as a GBS variant and nerve conduction studies in limbs were normal in this report. Preserved reflexes and even hyperreflexia may occur in patients with pure motor GBS and are not inconsistent with the diagnosis. It is more appropriate to classify this neuropathy as a GBS variant, which Capasso et al., suggest calling "acute motor conduction block neuropathy," emphasizing the presence of conduction blocks and avoiding the pathophysiologic implication that all conduction blocks are demyelinating in nature ${ }^{9}$.

In approximately two third of cases, it follows an infection; usually an upper respiratory viral type. Some pathogens are potential triggers of this serious neurological disease, including: Campylobacter jejuni, Mycoplasma pneumoniae and Cytomegalovirus. Although uncommon, hepatotrophic viruses (Hepatitis A, B, C and E virus) are increasingly recognized to be triggering microbes of $\mathrm{GBS}^{11}$.

Viruses are the most common organisms responsible for causeing GBS. Infections by $\mathrm{P}$. falciparum and P. Vivax ${ }^{12}$ have been documented to cause GBS in few sporadic cases, and further investigations (CSF, NCS) have confirmed the diagnosis. In our patient also the features of acute polyneuropathy developed after an acute attack of P. falciparum and therefore a causal relationship in this case is assumed. The exact pathogenesis of GBS following malaria infection is not known, but is likely to be immunogenic like that occurring after vaccination and viral or bacterial infections ${ }^{13}$. Another mechanism suggested for the development of polyneuropathy following a parasitic infection include parasitic emboli obstructing vasa nervosum thus causing anoxaemic stagnation in the vasa nervosum leading to temporary demyelination and recovery after disappearance of parasitaemia and establishment of normal blood flow in vasa nervosum ${ }^{14}$. Release of neurotoxins, associated metabolic and nutritional disturbance, immunemediated capillary damage, release of free radicals and tumour necrosis factor may also be responsible for the pathogenesis of GBS after P. falciparum infection ${ }^{15}$.

Autonomic dysfunction has been described in GBS and was noted in as many as $66 \%$ of patients in one study $^{16}$. Pathologic studies of the autonomic nervous system in GBS may demonstrate edema and inflammation of autonomic ganglia and destruction of peripheral ganglion cells. Chromatolysis, mononuclear cell infiltration, and nodules of Nageotte can be found within sympathetic ganglia ${ }^{17}$. Autoantibodies against gangliosides are often present, such as with anti-GM1 antibodies. Clinical manifestations of dysautonomia can range from seemingly innocuous profuse perspiration to life threatening arrhythmias.

Sir William Osler described a patient with GBS who died of "paralysis of the heart" ${ }^{18}$. Autonomic disturbance most commonly presents as sinus tachycardia, labile hypertension and postural hypotension. However, sinus bradycardia, asystole, supraventricular tachycardia, junctional tachycardia and ventricular tachycardia have also been reported. The risk of dysautonomia is higher in patients with quadriplegia, respiratory failure or bulbar involvemen ${ }^{19}$. Recent studies have 
indicated that serious bradyarrhythmias were observed even in less severely affected patients ${ }^{20}$. Bradyarrhythmias occur in up to $50 \%$ of patients with severe GBS and are due to parasympathetic overactivity $^{21}$.

Guillain-Barré syndrome is now the most frequent cause of acute flaccid paralysis worldwide and has a 'guarded' prognosis with up to $20 \%$ of patients experiencing severe long-term disability and up to $5 \%$ of patients dying even after receiving appropriate therapy ${ }^{22}$.

Diagnostic approach to GBS is based on clinical, laboratory, and electrophysiological criteria. The history is of paramount importance and is characterized by symmetric flaccid paresis associated with varying combinations of pain, paresthesia and numbness.

The National Institute of Neurological Disorders and Stroke (NINDS) has defined the weakness as progressive (over a four-week period) in their diagnostic criteria developed to guide physicians in making the diagnosis ${ }^{23}$. The Brighton Collaboration GBS Working Group ${ }^{24}$ has defined key clinical and epidemiologic features required for case definitions for GBS. The Working Group has stated in their case definition that limb weakness in GBS should be "bilateral and relatively symmetric" (generally with progression from legs to arms and bulbar muscles), associated with decreased or absent deep tendon reflexes in weak limbs, a monophasic illness pattern, an interval between onset and nadir of weakness between 12 hours and 28 days, subsequent clinical plateau, electrophysiologic findings consistent with GBS, cytoalbuminologic dissociation (ie elevation of cerebrospinal fluid (CSF) protein level above laboratory normal value, CSF total white cell count $<50$ cells $/ \mathrm{mm}$ and absence of an identified alternative diagnosis for weakness).

In Europe and North America, demyelinating GBS accounts for up to $90 \%$ of cases, but in other countries such as China and Japan, the axonal type GBS account for $30-65 \%$ of cases ${ }^{25}$. Early in the disease, electrophysiologic abnormalities are often mild or nonspecific. Motor conduction blocks
(CB) have been documented in only $2-15 \%$ of patients with GBS within 3 weeks from disease onset, and $\mathrm{CB}$ in intermediate nerve segments in the first days

of the disease is uncommon ${ }^{26}$. It is possible that mechanical impairment of the blood-nerve barrier at entrapment site may render these nerve segments more vulnerable to immunologic $\operatorname{attack}^{27}$.

The efficacy of plasmapheresis and intravenous immunoglobulin has been established in large international randomized trials and case reports, with corticosteroids proven ineffective ${ }^{28}$. Despite reported improvement with immunoglobulin therapy $^{29}$, the prognosis and recovery of AMSAN is generally poorer than the other subtypes and the main stay of treatment is supportive care ${ }^{30}$.

Poor prognostic factors include older age, rapid onset (less than seven days) prior to presentation, severe muscle weakness on admission, need for ventilatory support, an average distal motor response amplitude reduction to $<20 \%$ of normal, and preceding diarrhoeal illness ${ }^{31}$.

\section{CONCLUSION}

Generally Guillain-Barre syndrome (GBS) is a common cause of acute peripheral neuropathy and is characterized by hyporeflexia or areflexia. Hyperreflexia has been rarely reported with acute motor axonal neuropathy. This case reports highlights hyperreflexic guillain-barre syndrome as a rare presentation.

\section{REFERENCES}

1. Kuwabara S, Ogawara K, Koga M, Mori M, Hattori T, Yuki N. Hyperreflexia in Guillain- Barré syndrome: Relation with acute motor axonal neuropathy and antiGM1 antibody. J Neurol Neurosurg Psychiatry. 1999;67:180-4.

2. McKhann GM, Cornblath DR, Griffin JW, Ho TW, Li CY, Jiang Z, et al. Acute motor axonal neuropathy: A frequent cause of acute flaccid paralysis in China. Ann Neurol. 1993;33:333-42. 
3. Gupta D, Nair M, Baheti NN, Sarma PS, Kuruvilla A. Electrodiagnostic and clinical aspects of Guillain- Barré syndrome: An analysis of 142 cases. J Clin Neuromuscul Dis. 2008;10:42-51.

4. Somarajan A. Guillain Barre syndrome with brisk reflexes-Another variant. Neurol India. 2006;54:215-6.

5. Inés GS, Irene SG, Francisco JRR, Javier A. Guillain- Barré Syndrome: Natural history and prognostic factors: a retrospective review of 106 cases. BMC Neurology 2013; 13:95.

6. Asbury AK: Diagnostic considerations in Guillain-Barre syndrome. Ann Neurol 1981, 9(Suppl):1

7. Ropper AH: The Guillain-Barre syndrome. N Engl J Med 1992, 326(17):1130-1136.

8. Susuki K, Astumi M, Koga M, Hirata K, Yuki N. Acute facial diplegia and hyperreflexia- A Guillain Barre Syndrome variant. Neurology. 2004;62:825-7.

9. Capasso M, Caporale CM, Pomilio F, Gandolfi P, Lugaresi A, Uncini A. Acute motor conduction block neuropathy.Another Guillain- Barre syndrome variant. Neurology. 2003;61:617-22.

10. Van der Meché FG, Meulstee J, Vermeulen M, Kievit A. Patterns of conduction failure in the Guillain- Barré syndrome. Brain. 1988;111:405-16.

11. Jacobs BC, Rothbarth PH, van der Meché FG, Herbrink P, Schmitz PI, de Klerk MA, et al. The spectrum of antecedent infections in Guillain-Barre syndrome: A case-control study. Neurology 1998; 51:1110-5.

12. Sokrab TO, Eltahir A et al. Guillain-Barré syndrome following acute falciparum malaria. Neurology 2002; 59: 1281-3.

13. Schonberger LB, Hurwitz ES, Katona P et al. Guillain-Barré syndrome: it epidemiology and association with influenza vaccination. Ann Neurol 1981; 9 (suppl): 31-8.

14. Wijesundere A. Guillain-Barré syndrome in Plasmodium falciparum malaria. Postgrad Med J 1992; 68: 367-77.

15. Shubhakaran, Sharma CM. Acute inflammatory demyelinating polyneuropathy with $\mathrm{P}$. falciparum malaria. JAPI 2003; 51: 223-4.

16. Singh NK, Jaiswal AK, Misra S, Srivastava PK: Assessment of autonomic dysfunction in Guillain-Barre syndrome and its prognostic implications. Acta Neurol Scand 1987, 75(2):101-105.

17. Zochodne DW: Autonomic involvement in Guillain-Barre syndrome: a review. Muscle Nerve 1994, 17(10):1145-1155.

18. Osler W: The principles and practice of Medicine. New York. Appleton-CenturyCrofts; 1899.

19. Pfeiffer G, Schiller B, Kruse J, Netzer J: Indicators of dysautonomia in severe Guillain-Barre syndrome. J Neurol 1999, 246(11):1015-1022.

20. Flachenecker P, Toyka KV, Reiners K: Cardiac arrhythmias in Guillain-Barre syndrome. An overview of the diagnosis of a rare but potentially life-threatening complication. Nervenarzt 2001, 72(8):610-617.

21. Greenland P, Griggs RC: Arrhythmic complications in the Guillain-Barre syndrome. Arch Intern Med 1980, 140(8):1053-1055.

22. Yuki N, Hartung H. Review article: medical progress in Guillain Barre Syndrome. N Engl J Med 2012; 366: 2294-304.

23. National Institute of Neurological Disorders and Stroke. NINDS GuillainBarré syndrome information page. National Institute of Neurological Disorders and Stroke; 2011. Available from: www.ninds.gov/ disorders/gbs/gbs.htm 
24. Sejvar J, Kohl K, Gidudu J, Amato A, Bakshi N, Baxter R et al. Guillain-Barré syndrome and Fisher syndrome: case definitions and guidelines for collection, analysis, and presentation of immunization safety data. Vaccine 2011; 29: 599-612.

25. Seneviratne U. Guillain-Barré syndrome. Postgrad Med J 2000; 76: 774-82.

26. Gordon PH, Wilbourn AJ. Early electrodiagnostic findings in GuillainBarré syndrome. Arch Neurol. 2001;58:913-7.

27. Brown WF, Feasby TE. Conduction block and denervation in Guillain- Barré polyneuro- pathy. Brain. 1984;107:21939.

28. Rostásy KM, Huppke P, Beckers B, Brockmann K, Degenhardt V, Wesche B et al. Acute motor and sensory axonal neuropathy (AMSAN) in a 15-year-old boy presenting with severe pain and distal muscle weakness. Neuropediatrics 2005; 36: 260-4.

29. Hughes RA, Wijdicks EF, Benson E, Cornblath DR, Hahn AF, Meythaler JM et al. Supportive care for patients with Guillain-Barré syndrome. Arch Neurol 2005; 62: 1194-8.

30. Rees JH, Soudain SE, Gregson NA, Hughes RA. Campylobacter jejuni infection and Guillain-Barré syndrome. N Engl J Med 1995; 333: 1374-9.

31. Walgaard C, Lingsma HF, Ruts L, van Doorn PA, Steyerberg EW, Jacobs BC. Early recognition of poor prognosis in Guillain-Barre syndrome. Neurology 2011; 76: 968. 